\title{
TITEL
}

Katrin Höffler, Johannes Kaspar, Hendrik Schneider

\section{Editorial zum Titelthema „Lage und Zukunft der Kriminologie“}

Im Juni 2012 fand am Max-Planck-Institut für ausländisches und internationales Strafrecht in Freiburg die Tagung „Zur Lage der Kriminologie in Deutschland“ statt. Das diesbezügliche Memorandum in Gestalt der „Freiburger Erklärung“ deutscher Kriminologinnen und Kriminologen wurde uns dankenswerterweise von den Organisatoren zur Verfügung gestellt und ist in diesem Heft veröffentlicht. Zur Vertiefung der wichtigen Thematik und um eine breitere Diskussion anzuregen, haben wir Kriminologen aus Deutschland, Österreich und der Schweiz aus dem Kreis der Herausgeber der Neuen Kriminalpolitik und der Direktoren der kriminologischen Forschungsinstitute um ihre persönliche Meinung zu Lage und Zukunft der Kriminologie gebeten. Mit der Veröffentlichung dieser Statements wird im vorliegenden Heft begonnen. Weitere Beiträge gerade auch Stimmen außerhalb des Herausgeberkreises - sind uns herzlich willkommen!

Die überwiegend skeptischen Lagebilder weisen sicher zu Recht auf einen zunehmenden Bedeutungsverlust der Kriminologie an den Universitäten und nicht nur an den juristischen Fakultäten hin. Die Kriminologie sei allenfalls ein "juristisch geprägter Annex“ des Strafrechts und „vom Aussterben bedroht“. Hochschulkarrieren hätten nur dann Aussicht auf Erfolg, wenn die Bewerber das „Standbein“ in der Dogmatik und allenfalls das „Spielbein“ in der Kriminologie haben. Andererseits könne sich die empirische Forschung der deutschsprachigen Kriminologinnen und Kriminologen im internationalen Vergleich durchaus sehen lassen. Auch sei die deutschsprachige Kriminologie heute international besser sichtbar als in den 60er und 70er Jahren des 20. Jahrhunderts.

Diesen Zustandsbeschreibungen können wir uns im Ergebnis anschließen, auch wenn es gerade aus Augsburg, Leipzig und Tübingen positivere Stimmungsbilder zu vermelden gibt.

In Leipzig ist die Kriminologie nicht nur im Schwerpunktbereich Kriminalwissenschaften vertreten, sondern sie ist auch „Grundlagenfach“ und es kann ein „Grundlagenschein" erworben werden. Die zweistündige Vorlesung mit Abschlussklausur hat alleine im laufenden Wintersemester rund 500 Teilnehmerinnen und Teilnehmer. Im Rahmen der Ausbildung im Schwerpunktbereich bildet sich gegenwärtig mit Vorlesungen und Seminaren zu „Wirtschaftskriminologie und Compliance“ ein forschungs- und praxisnaher Veranstaltungszweig heraus, der von den Studierenden mit großem Interesse aufgenommen wird und die dogmatischen Fächer ergänzt und abrundet. Im „virtuellen 
Hörsaal“ (Videokonferenzen) fließen Inhalte aus dem Spektrum der Wirtschaftskriminologie zudem in Veranstaltungen mit der Law School der Universität Miami ein.

In Augsburg ist seit diesem Sommersemester die Vorlesung „Kriminologie“, die zuvor jahrelang nicht mehr angeboten worden war, wieder Teil des Curriculums des strafrechtlichen Schwerpunktbereichs - wenn auch nicht als Pflichtfach. Ein weiterer Ausbau der Kriminologie in Forschung und Lehre ist geplant, gerade auch im Hinblick auf wirtschaftskriminologische Themen.

In Tübingen hat die Kriminologie traditionsgemäß - aufgrund der Organisation als Institut - eine durchaus starke Stellung; das Lehrangebot ist umfassend. Dank der interdisziplinären Stellenbesetzung sind bspw. auch Kurse zu den Methoden der Kriminologie im Angebot. Die Vorlesungen zur Kriminologie werden nicht nur von den Juristen, sondern auch von Nebenfach-Studierenden, insbesondere aus den Erziehungswissenschaften, stark nachgefragt. Zudem ist ein Master-Studiengang in Kooperation mit anderen Universitäten und dem MPI Freiburg in Planung. Eine stärkere Zusammenarbeit mit Lehrstühlen aus den Bezugswissenschaften ist angestrebt.

Kriminologisches Hintergrundwissen ist durchaus auch auf dem Arbeitsmarkt gefragt, zum Beispiel wenn es um die Besetzung der Position eines Compliance Officers oder die Mitarbeit in den „forensic Abteilungen“ der Wirtschaftsprüfungsgesellschaften geht, so dass die Universitäten gut beraten sind, die entsprechende Ausbildung vorzuhalten und nicht abzubauen.

Wir sollten allerdings nicht erwarten, dass Strafrechtspflege und Wirtschaft auf die Kriminologie zugehen, sondern die Kriminologie muss sich mit Selbstbewusstsein einblenden und dort für zuständig erklären, wo sie traditionell zuständig ist. Dies gilt zum Beispiel auch für das Spektrum der Kriminalprognose. Hier hat sich die Kriminologie nicht gegen die Psychiatrie behaupten können, der „Markt“ wird fast vollständig von den medizinischen Sachverständigen dominiert. Es ist an der Zeit, den juristischen Kollegen zu transportieren, dass für bestimmte Fragen keine medizinische Fachkunde erforderlich ist, sondern der dementsprechend ausgebildete Kriminologe der eigentlich Sachverständige wäre. Auf diesem Weg würden auch originäre Berufsfelder für „den Kriminologen“ erschlossen.

Neue und spannende Forschungsfelder wie der Bereich der Transitional Justice, die Kriminalität im Internet oder auch Erkenntnisse zum menschlichen Verhalten aus den Neurowissenschaften sollten von den Kriminologen besetzt und nicht „kampflos“ den Bezugswissenschaften überlassen werden. Dasselbe gilt für die traditionell beratende Tätigkeit des Kriminologen bei kriminalpolitischen Gesetzesvorhaben; hierzu will die „Neue Kriminalpolitik“ weiterhin ihren Beitrag leisten. Sie bleibt auch im 25. Jahr ihres Bestehens und in neuem Format die kritische Stimme der Kriminologinnen und Kriminologen und Forum des Austauschs zwischen Wissenschaft und Praxis zu Gegenwartsund Grundlagenfragen. 